\title{
A POLYNOMIAL CHAOS APPROACH TO STOCHASTIC VARIATIONAL INEQUALITIES
}

\author{
RALF FORSTER AND RALF KORNHUBER
}

\begin{abstract}
We consider stochastic elliptic variational inequalities of the second kind involving a bilinear form with stochastic diffusion coefficient. We prove existence and uniqueness of weak solutions, propose a stochastic Galerkin approximation of an equivalent parametric reformulation, and show equivalence to a related collocation method. Numerical experiments illustrate the efficiency of our approach and suggest similar error estimates as for linear elliptic problems.
\end{abstract}

AMS classification: $65 \mathrm{~K} 15,65 \mathrm{~N} 30,65 \mathrm{~N} 35$

Keywords: Stochastic variational inequality, Karhunen-Loève expansion, polynomial chaos, finite elements, stochastic Galerkin method, stochastic collocation.

\section{INTRODUCTION}

Since the pioneering work of Ghanem and Spanos [12], spectral approximations in combination with stochastic Galerkin and collocation methods emerged as a new powerful approach to uncertainty propagation and quantification. Theoretical analysis mainly concentrated on linear elliptic problems [1, 2, 3, 4, 8, 11], but practical applications, e.g., in hydrology [24], meteorology [6], or fluid dynamics 20. underline the general relevance of this approach. We refer to the review article of Karniadakis et al. 17] and the recent monograph of Le Maître and Knio 19] for further information.

In this paper, we consider the stochastic variational inequality

$$
u \in X: \quad a(u, v-u)+\phi(v)-\phi(u) \geq \ell(v-u) \quad \forall v \in X,
$$

where the $X$-elliptic bilinear form $a(\cdot, \cdot)$ involves a stochastic diffusion coefficient $K(x, \omega)$. We prove existence and uniqueness for superposition operators $\phi$ satisfying certain growth conditions. Then we derive a parametric reformulation of (1.1) on the assumption that $K$ can be written in terms of a finite number of random variables. If necessary, suitable approximations can be obtained by well-known Karhunen-Loève expansions 7, 16, 22, 25]. The resulting parametric deterministic variational inequality is then approximated by a Galerkin method based on Lagrange polynomials. Inherent orthogonality properties of these ansatz functions provide the equivalence to corresponding collocation schemes [4]. Hence, the resulting algebraic problems can be efficiently solved by existing monotone multigrid methods [15, 18]. Numerical experiments confirm that the polynomial chaos approach clearly outperforms classical Monte Carlo methods for sufficiently smooth solutions and indicate that existing error estimates for stochastic elliptic equations could be extended to variational inequalities. This is the subject of ongoing research.

This work was supported by the Leibniz Gemeinschaft. 
The paper is organized as follows. In the following section, we give a precise formulation of the problem and show existence and uniqueness of a solution. Section [3] contains the parametric reformulation of the problem. Stochastic Galerkin methods and related collocation schemes are introduced in Section 4 In the final section, we consider a stochastic obstacle problem and a stochastic variational inequality from hydrology to numerically illustrate the convergence properties and the efficiency of our approach.

\section{Stochastic VARiational InEQUalities}

Let $D \subset \mathbb{R}^{d}$ denote a bounded domain with Lipschitz boundary and elements $x \in D$. For a given complete probability space $(\Omega, \mathcal{E}, P)$ with sample space $\Omega, \sigma$-algebra $\mathcal{E} \subset$ $2^{\Omega}$, and probability measure $P$, we consider the linear space $X=L^{2}\left(\Omega, \mathrm{d} P ; H_{0}^{1}(D)\right)$ of second-order random fields over the Hilbert space $H_{0}^{1}(D)$. More precisely, $X$ consists of all functions $v: \Omega \rightarrow H_{0}^{1}(D)$ such that $\|v\|_{H^{1}(D)}$ is $P$-measurable and

$$
E\left[\|v\|_{H^{1}(D)}^{2}\right]<\infty \text {. }
$$

Here, the Sobolev norm $\|v\|_{H^{1}(D)}$ is induced by the usual scalar product

$$
(v, w)_{H^{1}(D)}=(\nabla v \cdot \nabla w)_{L^{2}(D)}+(v, w)_{L^{2}(D)}, \quad(v, w)_{L^{2}(D)}=\int_{D} v(x) w(x) \mathrm{d} x
$$

and the expectation value $E[v]=\int_{\Omega} v(\omega) \mathrm{d} P$ is taken with respect to the probability measure $P$. Note that $X$ is a Hilbert space with scalar product and associated norm defined by

$$
(v, w)=E\left[(v, w)_{H^{1}(D)}\right], \quad\|v\|=E\left[\|v\|_{H^{1}(D)}^{2}\right]^{1 / 2},
$$

respectively. It is also convenient to introduce the $L^{2}$ analogue

$$
\|v\|_{0}=E\left[\|v\|_{L^{2}(D)}^{2}\right]^{1 / 2}
$$

of the $H^{1}$-like norm $\|\cdot\|$ and the data space $L^{\infty}\left(\Omega, \mathrm{d} P ; L^{\infty}(D)\right)$ equipped with $\|v\|_{\infty}=\operatorname{ess}_{(x, \omega) \in D \times \Omega}|v(x, \omega)|$.

We consider stochastic variational inequalities of the form (1.1) where the symmetric bilinear form $a(\cdot, \cdot)$,

$$
a(v, w)=E\left[\int_{D} K \nabla v \cdot \nabla w \mathrm{~d} x\right], \quad v, w \in X,
$$

involves the stochastic diffusion coefficient $K \in L^{\infty}\left(\Omega, \mathrm{d} P ; L^{\infty}(D)\right)$ satisfying

$$
0<K_{\min } \leq K(x, \omega) \leq K_{\max }<\infty \quad \text { a.e. in } D \times \Omega .
$$

The right-hand side $\ell \in X^{\prime}$ is given by

$$
\ell(v)=E\left[\int_{D} f v \mathrm{~d} x\right], \quad v \in X,
$$

where $f \in L^{2}(D)$ is a deterministic function, for simplicity. The superposition operator

$$
\phi(v)=E\left[\int_{D} \Phi(v(x, \cdot) \mathrm{d} x]\right.
$$

is induced by a scalar function $\Phi: \mathbb{R} \rightarrow \mathbb{R} \cup\{\infty\}$. We assume that $\Phi$ is convex and that the domain

$$
M=\{z \in \mathbb{R} \mid \Phi(z)<\infty\}
$$


of $\Phi$ is a closed interval with $0 \in M$. We additionally require that $\Phi$ is piecewise smooth in the sense that

$$
\Phi \in C^{2}\left(\theta_{i-1}, \theta_{i}\right), \quad \text { with } \quad\left|\Phi^{\prime \prime}(z)\right| \leq \text { const. } \forall z \in\left(\theta_{i-1}, \theta_{i}\right)
$$

holds on a partition

$$
-\infty \leq \inf M=\theta_{0}<\theta_{1}<\cdots<\theta_{N}<\theta_{N+1}=\sup M \leq+\infty
$$

of the interval $M$. Note that the growth condition in (2.4) is satisfied by piecewise quadratic functions occurring, e.g., in the enthalpy formulation of the Stefan problem 14], or by the generalized saturation resulting from Kirchhoff tranformation of the Richards equation describing saturated/unsaturated groundwater flow [5].

We will now state some properties of the functional $\phi$ as resulting from the above properties of $\Phi$.

Lemma 2.1. The functional $\phi: X \rightarrow \mathbb{R} \cup\{+\infty\}$ is convex, lower semicontinuous, and proper. The domain $\operatorname{dom} \phi=\{v \in X \mid \phi(v)<+\infty\} \subset X$ of $\phi$ is the non-empty, closed, and convex set

$$
\mathcal{M}=\{v \in X \mid v(x, \omega) \in M \text { a.e. in } D \times \Omega\},
$$

and $\phi$ is continuous on $\mathcal{M}$.

Proof. Let us first investigate the set $\mathcal{M}$. $\mathcal{M}$ is non-empty, because $0 \in M$ provides $0 \in \mathcal{M} . \mathcal{M}$ is convex, because $M$ is an interval and therefore convex. We now show that $\mathcal{M}$ is closed. Without loss of generality, let $M=(-\infty, 0]$ and consider a sequence $\left(v_{k}\right)_{k>0} \subset \mathcal{M}$ such that $v_{k} \rightarrow v, k \rightarrow \infty$ in $X$. We assume that $v \notin \mathcal{M}$. Then we can find a subset $D^{\prime} \times \Omega^{\prime} \subset D \times \Omega$ with positive measure such that $v(x, \omega)>0 \forall(x, \omega) \in D^{\prime} \times \Omega^{\prime}$. Using $v_{k} \in \mathcal{M}$, we get $v(x, \omega)-v_{k}(x, \omega) \geq v(x, \omega)>0$, a.e. in $D^{\prime} \times \Omega^{\prime}$. This leads to

$$
\left\|v-v_{k}\right\|^{2} \geq \int_{\Omega^{\prime}}\left\|v-v_{k}\right\|_{L^{2}\left(D^{\prime}\right)}^{2} \mathrm{~d} P \geq \int_{\Omega^{\prime}}\|v\|_{L^{2}\left(D^{\prime}\right)}^{2} \mathrm{~d} P>0 \quad \forall k \geq 0
$$

in contradiction to $v_{k} \rightarrow v$ in $X$. In the next step, we show $\mathcal{M}=\operatorname{dom} \phi$. Let $v \in \mathcal{M}$. Then $|\Phi(v(x, \omega))| \leq c\left(1+|v(x, \omega)|^{2}\right)$ holds with some $c>0$ due to the growth condition in (2.4). Hence,

$$
|\phi(v)| \leq c\left(1+E\left[\|v\|_{L^{2}(D)}^{2}\right]\right) \leq C\left(1+\|v\|^{2}\right)<\infty
$$

and therefore $v \in \operatorname{dom} \phi$. As $v \notin \mathcal{M}$ implies $\phi(v)=\infty$, we have shown dom $\phi=\mathcal{M}$.

In order to demonstrate that $\phi$ is continuous on $\mathcal{M}$, assume that $\left(v_{k}\right)_{k \geq 0} \subset \mathcal{M}$ converges to $v$ in $X$. As $\mathcal{M}$ is closed, we have $v \in \mathcal{M}$. Utilizing (2.4), we find

$$
\left|\Phi(v(x, \omega))-\Phi\left(v_{k}(x, \omega)\right)\right| \leq c\left(1+|v(x, \omega)|+\left|v_{k}(x, \omega)\right|\right)\left|v(x, \omega)-v_{k}(x, \omega)\right|
$$

a.e. in $D \times \Omega$ with some $c$ independent of $v(x, \omega)$ and $v_{k}(x, \omega)$. After integrating over $D \times \Omega$, the Cauchy-Schwarz inequality provides

$$
\begin{aligned}
\left|\phi(v)-\phi\left(v_{k}\right)\right| & \leq c \int_{\Omega} \int_{D}\left(1+|v(x, \omega)|+\left|v_{k}(x, \omega)\right|\right)\left|v(x, \omega)-v_{k}(x, \omega)\right| \mathrm{d} x \mathrm{~d} P \\
& \leq \sqrt{3} c E\left[1+\|v\|_{L^{2}(D)}^{2}+\left\|v_{k}\right\|_{L^{2}(D)}^{2}\right]^{1 / 2}\left\|v-v_{k}\right\|_{0}
\end{aligned}
$$

As $E\left[\left\|v_{k}\right\|_{L^{2}(D)}^{2}\right] \leq\left\|v_{k}\right\|^{2}$ is bounded, this leads to $\left|\phi(v)-\phi\left(v_{k}\right)\right| \rightarrow 0$ for $k \rightarrow \infty$.

The convexity of $\Phi$ implies that $\phi$ is convex. We now show that $\phi$ is lower semicontinuous. Let $v_{k} \rightarrow v, k \rightarrow \infty$, in $X$. Assume that for each $k_{0} \in \mathbb{N}$, there is an index $k \geq k_{0}$ such that $v_{k} \in \mathcal{M}$. Then we can find a subsequence $\left(v_{k_{i}}\right)_{i \geq 0} \subset \mathcal{M}$ still converging to $v$. As $\mathcal{M}$ is closed this implies $v \in \mathcal{M}$ and the continuity of $\phi$ on $\mathcal{M}$ yields $\phi\left(v_{k_{i}}\right) \rightarrow \phi(v)$. As the same holds true for any subsequence contained 
in $\mathcal{M}$ and $\phi\left(v_{k}\right)=+\infty$ for $v_{k} \notin \mathcal{M}$, we get $\liminf _{k \rightarrow \infty} \phi\left(v_{k}\right)=\phi(v)$. In the remaining case, $v_{k} \notin \mathcal{M} \forall k \geq k_{0}$ holds with some fixed $k_{0} \geq 0$. Then we clearly have $\liminf \operatorname{in}_{k \rightarrow \infty} \phi\left(v_{k}\right)=\infty \geq \phi(v)$. From $\mathcal{M} \neq \emptyset$ and $\phi(v) \in \mathbb{R} \cup\{+\infty\}$ we get $\phi \not \equiv \infty$ and $\phi(v)>-\infty \forall v \in X$ so that $\phi$ is proper. This concludes the proof.

In contrast to the deterministic case, we do not obtain the weak lower semicontinuity of $\phi$, because weak convergence in $X$ does not imply strong convergence with respect to the $L^{2}$ norm $\|\cdot\|_{0}$. Hence, the existing abstract convergence theory 13 , Section I.6] cannot be applied to finite element discretizations as described below.

We are now ready to state the main result of this section.

Theorem 2.2. The variational inequality (1.1) has a unique solution $u \in X$ and is equivalent to the minimization problem

$$
u \in X: \quad \mathcal{J}(u)+\phi(u) \leq \mathcal{J}(v)+\phi(v) \quad \forall v \in X
$$

with the quadratic functional $\mathcal{J}(v)=\frac{1}{2} a(v, v)-\ell(v)$.

Proof. The equivalence of (2.5) and the variational inequality (1.1) follows from 9, Proposition 2.2]. As $a(\cdot, \cdot)$ is $X$-elliptic by (2.2), $\ell \in X^{\prime}$ by (2.3), and $\phi$ is convex, lower semicontinuous and proper, by Lemma 2.1 the functional $\mathcal{J}+\phi$ is strictly convex, coercive, lower semicontinuous, and proper on $X$. Therefore, the minimization problem (2.5) has a unique solution (cf. 9, Proposition 1.2]).

\section{Stochastic Parameters}

3.1. Karhunen-Loève expansion. The goal of this subsection is a suitable approximation of the diffusion coefficient in terms of a finite number of random variables. Let

$$
\bar{K}(x)=E[K(x, \cdot)] \leq K_{\max }
$$

denote the expectation value of $K(x, \omega)$. We consider the eigenfunctions $g_{r}$ of the symmetric, positive definite covariance operator $\mathcal{C}_{K}: L^{2}(D) \rightarrow L^{2}(D)$ defined by

$$
\mathcal{C}_{K} v(x)=\int_{D} C_{K}(x, y) v(y) d y,
$$

with kernel function

$$
C_{K}(x, y)=E[(K(x, \cdot)-\bar{K}(x))(K(y, \cdot)-\bar{K}(y))],
$$

and the associated eigenvalues $\lambda_{r}>0$. The eigenfunctions $g_{r}$ constitute an orthonormal basis of $L^{2}(D)$. The well-known Karhunen-Loève expansion 16, 22]

$$
K(x, \omega)-\bar{K}(x)=\sum_{r=1}^{\infty} \sqrt{\lambda_{r}} g_{r}(x) \xi_{r}(\omega),
$$

is the Fourier expansion of the random function $K(\cdot, \omega)-\bar{K}$ into $g_{r}$. The Fourier coefficients

$$
\xi_{r}(\omega)=\frac{1}{\sqrt{\lambda_{r}}} \int_{D}(K(x, \omega)-\bar{K}(x)) g_{r}(x) \mathrm{d} x
$$

are uncorrelated random variables with zero mean and unit variance, i.e.

$$
E\left[\xi_{r}\right]=0, \quad E\left[\xi_{r} \xi_{s}\right]=\delta_{r s} \quad(\text { Kronecker } \delta), \quad r, s=1, \ldots .
$$

Convergence takes place in $L^{2}\left(\Omega, \mathrm{d} P ; L^{2}(D)\right)$ [25, Theorem 2.5].

We consider the finite dimensional approximation

$$
K_{R}(x, \omega)=\bar{K}(x)+\sum_{r=1}^{R} \sqrt{\lambda_{r}} g_{r}(x) \xi_{r}(\omega) \approx K(x, \omega)
$$


with some fixed $R \in \mathbb{N}$. This approximation is optimal in the sense that $K_{R}$ is minimizing the $L^{2}$ error $\left\|K-K_{R}\right\|_{0}$ over $\operatorname{span}\left\{g_{r} \xi_{r} \mid r=1, \ldots, R\right\}[12]$. Obviously, we have $K_{R} \rightarrow K, R \rightarrow \infty$, in $L^{2}\left(\Omega, \mathrm{d} P ; L^{2}(D)\right)$. The decay of the eigenvalues $\lambda_{r}$ and thus the convergence speed strongly depends on the regularity of the covariance kernel function $C_{K}$. For example, if $C_{K}$ is piecewise analytic (like Gaussian covariance), then we have exponential decay $0<\lambda_{r} \leq c_{1} \exp \left(-c_{2} r^{1 / d}\right)$ with constants $c_{1}$, $c_{2}>0$ independent of $r$. For a proof and further information we refer to [17, 25] and the literature cited therein.

The approximation $K_{R}$ of $K$ gives rise to the approximate bilinear form

$$
a_{R}(v, w)=E\left[\int_{D} K_{R} \nabla v \cdot \nabla w \mathrm{~d} x\right], \quad v, w \in X .
$$

In order to make sure that $a_{R}(\cdot, \cdot)$ is still $X$-elliptic, we impose the additional assumption on $K$ that

$$
\text { the random variables } \xi_{r}, r=1, \ldots, R \text {, are independent, }
$$

which means that $P\left(\bigcap_{k=1}^{n}\left\{\xi_{r_{k}} \in A_{r_{k}}\right\}\right)=\prod_{k=1}^{n} P\left(\left\{\xi_{r_{k}} \in A_{r_{k}}\right\}\right)$ holds for every finite collection $\xi_{r_{1}}, \ldots, \xi_{r_{n}}$ and Borel sets $A_{r_{1}}, \ldots, A_{r_{n}}$ with $1 \leq r_{1}<\cdots<r_{n} \leq R$. Note that (3.5) follows from (3.2), if the random variables $\xi_{r}$ are Gaussian. Now let $\mathcal{E}_{R}=\sigma\left(\xi_{1}, \ldots, \xi_{R}\right) \subset \mathcal{E}$ denote the $\sigma$-algebra generated by the random variables $\xi_{1}, \ldots, \xi_{R}$. Then it has been shown in [26] utilizing assumption [3.5], that

$$
K_{R}(x, \omega)=E\left[K(x, \cdot) \mid \mathcal{E}_{R}\right]
$$

holds (conditional expectations). Then the desired ellipticity condition

$$
0<K_{\min } \leq K_{R}(x, \omega) \leq K_{\max }<\infty \quad \text { a.e. in } D \times \Omega
$$

follows from (2.2) and the monotonicity of conditional expectations. Note that (3.6) holds uniformly in $R$.

3.2. Parametric deterministic formulation. In light of the considerations of the previous subsection, we assume from now on that the diffusion coefficient $K(x, \omega) \in L^{\infty}\left(\Omega, \mathrm{d} P ; L^{\infty}(D)\right) \subset L^{2}\left(\Omega, \mathrm{d} P ; L^{2}(D)\right)$ can be written in the form

$$
K(x, \omega)=\bar{K}(x)+\sum_{r=1}^{R} \alpha_{r}(x) \xi_{r}(\omega)
$$

with $\alpha_{r} \in L^{2}(D)$ and random variables $\xi_{r}: \Omega \rightarrow \mathbb{R}, r=1, \ldots, R$. Then, by means of the Doob-Dynkin lemma [23, Lemma 2.1.2], the solution

$$
u(x, \omega)=\tilde{u}\left(x, \xi_{1}(\omega), \ldots, \xi_{R}(\omega)\right)
$$

of the stochastic variational inequality (1.1) depends only on $x$ and the random variables $\xi=\left(\xi_{1}, \ldots, \xi_{R}\right)$. As a consequence, we can reformulate (1.1) as a parametric deterministic problem for $\tilde{u}: D \times \xi(\Omega) \rightarrow \mathbb{R}$. To this end, let $I_{r}=\xi_{r}(\Omega) \subset \mathbb{R}$ denote the range of $\xi_{r}, r=1, \ldots, R$. Then $\xi(\Omega)=I=I_{1} \times \cdots \times I_{R} \subset \mathbb{R}^{R}$ equipped with the $\sigma$-algebra $\operatorname{Bor}(I)$ and a unique probability measure $P_{\xi}$ satisfying

$$
P_{\xi}=\prod_{r=1}^{R} P_{\xi_{r}}, \quad \int_{A} \mathrm{~d} P_{\xi_{r}}=\int_{\xi_{r}^{-1}(A)} \mathrm{d} P, \quad A \in \operatorname{Bor}(I)
$$

is a probability space. We assume that the random variables $\xi_{r}$ have the density functions $\operatorname{pdf}_{r}: I_{r} \rightarrow \mathbb{R}_{+}$with $\operatorname{pdf}_{r} \in L^{\infty}\left(I_{r}\right)$. Then

$$
\operatorname{pdf}(\mathbf{y})=\prod_{r=1}^{R} \operatorname{pdf}_{r}\left(y_{r}\right), \quad \mathbf{y}=\left(y_{1}, \ldots, y_{R}\right) \in I
$$


is the probability density function of $\xi$. As a consequence, the expectation value of a random variable $v(\xi(\cdot)): \Omega \rightarrow \mathbb{R}$ can be rewritten as

$$
E[v]=\int_{\Omega} v(\xi(\omega)) \mathrm{d} P(\omega)=\int_{I} v(\mathbf{y}) \operatorname{pdf}(\mathbf{y}) \mathrm{d} \mathbf{y} .
$$

Exploiting (3.7) and (3.9), the stochastic variational inequality (1.1) can be equivalently rewritten as the parametrized deterministic problem

$$
\tilde{u} \in Y: \quad \tilde{a}(\tilde{u}, v-\tilde{u})+\tilde{\phi}(v)-\tilde{\phi}(\tilde{u}) \geq \tilde{\ell}(v-\tilde{u}) \quad \forall v \in Y
$$

on the Hilbert space $Y=L^{2}\left(I, \mathrm{~d} P_{\xi} ; H_{0}^{1}(D)\right)$ by replacing the random variables $\xi=\left(\xi_{1}, \ldots, \xi_{R}\right)$ by coordinates $\mathbf{y}=\left(y_{1}, \ldots, y_{R}\right)$. The functionals $\tilde{\phi}, \tilde{\ell}$, and the $Y$-elliptic bilinear form $\tilde{a}(\cdot, \cdot)$ induced by

$$
\tilde{K}\left(x, y_{1}, \ldots, y_{R}\right)=\bar{K}(x)+\sum_{r=1}^{R} \alpha_{r}(x) y_{r}
$$

are obtained by integrating over $I$ with respect to $\mathrm{d} P_{\xi}=\operatorname{pdf}(\mathbf{y}) \mathrm{d} \mathbf{y}$. For ease of notation the superscript ${ }^{\sim}$ is mostly skipped in the sequel. We now concentrate on the approximation of the parametric problem (3.10).

\section{Discretization}

4.1. Polynomial chaos. It is well-known [2, 3] that the (approximate) solution space $Y=L^{2}\left(I, \mathrm{~d} P_{\xi} ; H_{0}^{1}(D)\right)$ has a tensor product structure in the sense that functions $v \in Y$ can be written as infinite product expansions

$$
v(x, \mathbf{y})=\sum_{\nu} v_{\nu}(x) w_{\nu}(\mathbf{y})
$$

with $v_{\nu} \in H_{0}^{1}(D)$ and $w_{\nu} \in L^{2}\left(I, \mathrm{~d} P_{\xi}\right)$. For a Ritz-Galerkin approximation of the parametric problem (3.10) we therefore consider a subspace $Y_{\mathcal{Q}} \subset Y$ consisting of functions of the form

$$
v(x, \mathbf{y})=\sum_{\nu \in \mathcal{Q}} v_{\nu}(x) \Psi_{\nu}(\mathbf{y}), \quad x \in D, \mathbf{y}=\left(y_{1}, \ldots, y_{R}\right) \in I=I_{1} \times \cdots \times I_{R},
$$

with coefficients $v_{\nu} \in H_{0}^{1}(D)$, orthogonal polynomials $\Psi_{\nu}$,

$$
\int_{I} \Psi_{\nu}(\mathbf{y}) \Psi_{\mu}(\mathbf{y}) \operatorname{pdf}(\mathbf{y}) \mathrm{d} \mathbf{y}=\delta_{\nu, \mu}, \quad \nu, \mu \in \mathcal{Q},
$$

and a suitable index set $\mathcal{Q}$ to be specified below. This approach is often called polynomial chaos [12, 27]. In light of (3.8), we choose the polynomials with tensor product structure

$$
\Psi_{\nu}(\mathbf{y})=\Psi_{\left(\nu_{1}, \ldots, \nu_{R}\right)}\left(y_{1}, \ldots, y_{R}\right)=\prod_{r=1}^{R} \psi_{\nu_{r}}\left(y_{r}\right)
$$

consisting of scalar polynomials $\psi_{\nu_{r}}$ of maximal degree $m_{r}, r=1, \ldots, R$. We select the scalar Lagrange polynomials

$$
\psi_{\nu_{r}}(y)=\frac{1}{\sqrt{\eta_{\nu_{r}}}} \prod_{\substack{\pi \in Q_{r} \\ \pi \neq \nu_{r}}} \frac{y-\pi}{\nu_{r}-\pi}, \quad \nu_{r} \in Q_{r},
$$

and let $Q_{r}$ denote the set of $m_{r}+1$ Gaussian quadrature points $\nu_{r} \in I_{r}$ with associated positive weights $\eta_{\nu_{r}}$. The orthogonality (4.2) then follows from

$$
\int_{I_{r}} \psi_{\nu_{r}}(y) \psi_{\mu_{r}}(y) \operatorname{pdf}_{r}(y) \mathrm{d} y=\sum_{\pi \in Q_{r}} \psi_{\nu_{r}}(\pi) \psi_{\mu_{r}}(\pi) \eta_{\pi}=\delta_{\nu_{r}, \mu_{r}}, \quad \nu_{r}, \mu_{r} \in Q_{r}
$$


with the set $\mathcal{Q}=Q_{1} \times \cdots \times Q_{R}$ of multiindices $\nu=\left(\nu_{1}, \ldots, \nu_{R}\right)$. Finally, 4.5 is a consequence of the exact integrability of polynomials with maximal degree $2 m_{r}+1$ and of the Lagrange property

$$
\psi_{\nu_{r}}(\pi)=\frac{1}{\sqrt{\eta_{\pi}}} \delta_{\nu_{r}, \pi}, \quad \nu_{r}, \pi \in Q_{r}
$$

Our particular choice of polynomials is motivated by the following proposition.

Proposition 4.1. For given $v, w \in H_{0}^{1}(D)$ the functions $v \Psi_{\nu}, w \Psi_{\mu}$ are a-orthogonal in the sense that

$$
a\left(v \Psi_{\nu}, w \Psi_{\mu}\right)=(\bar{K} \nabla v, \nabla w)_{L^{2}(D)}+\delta_{\nu, \mu} \sum_{r=1}^{R} \nu_{r}\left(\alpha_{r} \nabla v, \nabla w\right)_{L^{2}(D)}
$$

holds for all $\nu, \mu \in \mathcal{Q}$.

Proof. From the definition (3.11) of $K=\tilde{K}$ and of the associated bilinear form $a(\cdot, \cdot)=\tilde{a}(\cdot, \cdot)$ and the orthogonality (4.2), we have

$$
\begin{aligned}
& a\left(v \Psi_{\nu}, w \Psi_{\mu}\right)=(\bar{K} \nabla v, \nabla w)_{L^{2}(D)} \\
&+\sum_{r=1}^{R}\left(\alpha_{r} \nabla v, \nabla w\right)_{L^{2}(D)} \int_{I} y_{r} \Psi_{\nu}(\mathbf{y}) \Psi_{\mu}(\mathbf{y}) \operatorname{pdf}(\mathbf{y}) \mathrm{d} \mathbf{y} .
\end{aligned}
$$

Then, the exactness of scalar Gauß quadrature for polynomials with maximal degree $2 m_{r}+1$ and the scalar orthogonality (4.5) yields

$$
\begin{aligned}
& \int_{I} y_{r} \Psi_{\nu}(\mathbf{y}) \Psi_{\mu}(\mathbf{y}) \operatorname{pdf}(\mathbf{y}) \mathrm{d} \mathbf{y} \\
= & \int_{I_{r}} y_{r} \psi_{\nu_{r}}(y) \psi_{\mu_{r}}(y) \operatorname{pdf}_{r}(y) \mathrm{d} y \cdot \prod_{\substack{s=1 \\
s \neq r}}^{R} \int_{I_{s}} \psi_{\nu_{s}}(y) \psi_{\mu_{s}}(y) \operatorname{pdf}_{s}(y) \mathrm{d} y \\
= & \sum_{\pi \in Q_{r}} \pi \psi_{\nu_{r}}(\pi) \psi_{\mu_{r}}(\pi) \eta_{\pi} \cdot \prod_{\substack{s=1 \\
s \neq r}}^{R} \delta_{\nu_{s}, \mu_{s}}=\nu_{r} \delta_{\nu, \mu} .
\end{aligned}
$$

This concludes the proof.

According to Proposition 4.1 the Ritz-Galerkin approximation based on the subspace $Y_{\mathcal{Q}}$ with tensor product polynomials $\Psi_{\nu}$ spanned by the Lagrange basis leads to completely decoupled problems for the unknown coefficients $u_{\nu} \in H_{0}^{1}(D)$. Each of these problems is obtained by setting $\mathbf{y}=\nu \in \mathcal{Q}$. This relates our approach to stochastic collocation methods [4, 21, 28].

Corollary 4.2. The Ritz-Galerkin approximation of 3.10 induced by the subspace $Y_{\mathcal{Q}}$ is equivalent to collocation in the multivariate Gauß points $\nu \in \mathcal{Q}$.

As an immediate consequence of Corollary 4.2 the convergence analysis for Galerkin methods 2] carries over to collocation methods [4] and vice versa.

4.2. Finite elements. For simplicity, we assume that $D$ has polygonal (polyhedral) boundary. Then $\mathcal{T}$ denotes a partition of $D$ into simplices $t \in \mathcal{T}$ with minimal diameter $h$ and interior vertices $\mathcal{N}$. We assume that $\mathcal{T}$ is regular in the sense that the intersection of two simplices $t, t^{\prime} \in \mathcal{T}$ either consists of a common lower dimensional simplex or is empty. The finite element space $\mathcal{S} \subset H_{0}^{1}(D)$ consisting of all 
continuous functions which are linear on all $t \in \mathcal{S}$ is spanned by the nodal basis $\varphi_{p}$, $p \in \mathcal{N}$, defined by

$$
\varphi_{p} \in \mathcal{S}, \quad \varphi_{p}(q)=\delta_{p, q}, \quad p, q \in \mathcal{N} .
$$

Replacing $H_{0}^{1}(D)$ by $\mathcal{S}$, we obtain the finite dimensional subspace

$$
Y_{\mathcal{S} \mathcal{Q}}=\left\{v \in Y \mid v(x, \mathbf{y})=\sum_{\nu \in \mathcal{Q}} v_{\nu}(x) \Psi_{\nu}(\mathbf{y}), v_{\nu} \in \mathcal{S}\right\} \subset Y_{\mathcal{Q}} \subset Y
$$

spanned by the basis $\varphi_{p} \Psi_{\nu}, p \in \mathcal{N}, \nu \in \mathcal{Q}$ with orthogonal polynomials $\Psi_{\nu}$ defined in (4.3) and (4.4). As usual, we further approximate the functional

$$
\phi(v)=\int_{I} \int_{D} \Phi(v(x, \mathbf{y})) \mathrm{d} x \operatorname{pdf}(\mathbf{y}) \mathrm{d} \mathbf{y}, \quad v \in Y,
$$

by lumping, or, more precisely, by the pointwise approximation

$$
\phi_{\mathcal{S} \mathcal{Q}}(v)=\sum_{\nu \in \mathcal{Q}} \sum_{p \in \mathcal{N}} \Phi(v(p, \nu)) h_{p} \eta_{\nu}, \quad h_{p}=\int_{D} \varphi_{p} \mathrm{~d} x, \quad v \in Y_{\mathcal{S} \mathcal{Q}},
$$

based on piecewise linear approximation in $x$ and Gauß quadrature in $\mathbf{y}$. The consistency of this approximation is shown in [10, Section 3.3]. We now state that this discretization preserves the essential properties of $\phi$.

Lemma 4.3. The functional $\phi_{\mathcal{S Q}}: Y_{\mathcal{S Q}} \rightarrow \mathbb{R} \cup\{+\infty\}$ is convex, lower semicontinuous and proper. The domain of $\phi_{\mathcal{S} \mathcal{Q}}$ is the non-empty, closed and convex set

$$
\mathcal{M}_{\mathcal{S Q}}=\left\{v \in Y_{\mathcal{S Q}} \mid v(p, \nu) \in M, \forall p \in \mathcal{N}, \nu \in \mathcal{Q}\right\}
$$

and $\phi_{\mathcal{S} \mathcal{Q}}$ is continuous on $\mathcal{M}_{\mathcal{S Q}}$.

Proof. The assumptions on the scalar function $\Phi$ as stated in Section 2 imply that $\Phi$ is convex, lower semicontinuous, proper and continuous on its domain $M$. These properties carry over to the multivariate sum of scalar functions $\phi_{\mathcal{S} \mathcal{Q}}$.

We are now ready to state a discrete counterpart of Theorem 2.2

Theorem 4.4. The discrete variational inequality

$u_{\mathcal{S} \mathcal{Q}} \in Y_{\mathcal{S} \mathcal{Q}}: \quad a\left(u_{\mathcal{S} \mathcal{Q}}, v-u_{\mathcal{S} \mathcal{Q}}\right)+\phi_{\mathcal{S} \mathcal{Q}}(v)-\phi_{\mathcal{S} \mathcal{Q}}\left(u_{\mathcal{S} \mathcal{Q}}\right) \geq \ell\left(v-u_{\mathcal{S} \mathcal{Q}}\right) \quad \forall v \in Y_{\mathcal{S} \mathcal{Q}}$ has a unique solution $u_{\mathcal{S} \mathcal{Q}}$ and is equivalent to the minimization problem

$$
u_{\mathcal{S Q}} \in Y_{\mathcal{S Q}}: \quad \mathcal{J}\left(u_{\mathcal{S Q}}\right)+\phi_{\mathcal{S} \mathcal{Q}}\left(u_{\mathcal{S} \mathcal{Q}}\right) \leq \mathcal{J}(v)+\phi_{\mathcal{S} \mathcal{Q}}(v) \quad \forall v \in Y_{\mathcal{S Q}}
$$

with the quadratic functional $\mathcal{J}(v)=\frac{1}{2} a(v, v)-\ell(v)$.

We emphasize that the Ritz-Galerkin method (4.8) is equivalent to a collocation method.

Theorem 4.5. The unknown coefficients $u_{\nu}$ of $u_{\mathcal{S} \mathcal{Q}}=\sum_{\nu \in \mathcal{Q}} u_{\nu} \Psi_{\nu}$ are the unique solutions of the finite element minimization problems

$$
u_{\nu} \in \mathcal{S}: \quad \mathcal{J}_{\nu}\left(u_{\nu}\right)+\phi_{\nu}\left(u_{\nu}\right) \leq \mathcal{J}_{\nu}(v)+\phi_{\nu}(v) \quad \forall v \in \mathcal{S} .
$$

For each $\nu \in \mathcal{Q}$ the quadratic functional $\mathcal{J}_{\nu}=\frac{1}{2} a_{\nu}(\cdot, \cdot)-\ell_{\nu}$ consists of the $\mathcal{S}$-elliptic bilinear form

$$
a_{\nu}(v, w)=(\bar{K} \nabla v, \nabla w)_{L^{2}(D)}+\sum_{r=1}^{R} \nu_{r}\left(\alpha_{r} \nabla v, \nabla w\right)_{L^{2}(D)},
$$

and the linear functional $\ell_{\nu}=(f, \cdot)_{L^{2}(D)} \sqrt{\eta}_{\nu} \in \mathcal{S}^{\prime}$ with weights $\eta_{\nu}=\eta_{\nu_{1}} \cdots \eta_{\nu_{R}}$. The functional $\phi_{\nu}: \mathcal{S} \rightarrow \mathbb{R} \cup\{+\infty\}$ is defined by

$$
\phi_{\nu}(v)=\sum_{p \in \mathcal{N}} \Phi\left(v(p) / \sqrt{\eta_{\nu}}\right) h_{p} \eta_{\nu}
$$


Proof. The $\mathcal{S}$-ellipticity of $a_{\nu}(\cdot, \cdot)$ follows from

$$
a_{\nu}(v, w)=a\left(v \Psi_{\nu}, w \Psi_{\nu}\right), \quad v, w \in \mathcal{S}, \quad \nu \in \mathcal{Q}
$$

and the $X$-ellipticity of $a(\cdot, \cdot)$. Obviously, $\ell_{\nu} \in \mathcal{S}^{\prime}$ and $\phi_{\nu}$ is convex, lower semicontinuous and proper so that [4.9) is uniquely solvable. Exploiting the orthogonality as stated in Proposition 4.1 and the pointwise approximation 4.7] of $\phi$, we obtain

$$
\mathcal{J}(v)+\phi_{\mathcal{S} \mathcal{Q}}(v)=\sum_{\nu \in \mathcal{Q}} \mathcal{J}_{\nu}\left(v_{\nu}\right)+\phi_{\nu}\left(v_{\nu}\right) \quad \forall v=\sum_{\nu \in \mathcal{Q}} v_{\nu} \Psi_{\nu} \in Y_{\mathcal{S} \mathcal{Q}}
$$

which concludes the proof.

We emphasize that the minimization problems 4.9 can be solved efficiently by monotone multigrid methods [15, 18]. This property supports our choice of Lagrangian ansatz functions. Other selections providing the same approximation properties at less degrees of freedom [26] typically lead to strongly coupled algebraic problems which (at the moment) cannot be solved with comparable efficiency.

In the linear case $\phi \equiv 0$, discretization error estimates

$$
\left\|u-u_{\mathcal{S} \mathcal{Q}}\right\| \leq \mathcal{O}\left(h+\sum_{r=1}^{R} \beta^{m_{r}+1}\right)
$$

with $0<\beta<1$ have been shown separately for the Ritz-Galerkin method (4.8) and for the collocation method (4.9) by Babuška et al. [2] and Babuška et al. [4], respectively. Furthermore, the convergence of the expectation of the solution can be estimated as

$$
\left\|E\left[u-u_{\mathcal{S} \mathcal{Q}}\right]\right\|_{L^{2}(D)} \leq \mathcal{O}\left(h^{2}+\sum_{r=1}^{R} \beta^{2 m_{r}+2}\right)
$$

with $\beta$ as above according to [2]. In our numerical computations to be reported in the next section, we observed similar convergence results for the variational inequality (4.8). Theoretical verification will be the subject of future research.

\section{NumericAl EXPERIMENTS}

5.1. A stochastic obstacle problem. We consider the stochastic variational inequality (1.1) with $\phi$ generated by the characteristic function $\Phi=\chi_{[0, \infty)}$ of $[0, \infty)$. In this case, (1.1) takes the form of a stochastic obstacle problem

$$
u \in \mathcal{M}: \quad a(u, v-u) \geq \ell(v-u) \quad \forall v \in \mathcal{M}
$$

with $\mathcal{M}=\{v \in X \mid v(x, \omega) \geq 0$ a.e. in $D \times \Omega\}$. We select $D=(-1,1) \times(-1,1)$ and the diffusion coefficient

$$
K(x, \omega)=1+\frac{\cos |x|^{2}}{10} \xi_{1}(\omega)+\frac{\sin |x|^{2}}{10} \xi_{2}(\omega)
$$

with uniformly distributed random variables $\xi_{1}, \xi_{2}, l(v)=E\left[\int_{D} f v \mathrm{~d} x\right]$ with

$$
f(x, \omega)=\left\{\begin{array}{cl}
-8 e^{2\left(\xi_{1}(\omega)+\xi_{2}(\omega)\right)\left(K(x, \omega) \cdot\left(2|x|^{2}-r^{2}\right)\right.} & |x|>r \\
\left.+\left(|x|^{2}-r^{2}\right)|x|^{2}\left(-\frac{\sin |x|^{2}}{10} \xi_{1}(\omega)+\frac{\cos |x|^{2}}{10} \xi_{2}(\omega)\right)\right), & , \\
4 r^{2} e^{2\left(\xi_{1}(\omega)+\xi_{2}(\omega)\right)}\left(2 K(x, \omega) \cdot\left(-1-r^{2}+|x|^{2}\right)\right. & |x| \leq r \\
\left.+\left(-2-2 r^{2}+|x|^{2}\right)|x|^{2}\left(-\frac{\sin |x|^{2}}{10} \xi_{1}(\omega)+\frac{\cos |x|^{2}}{10} \xi_{2}(\omega)\right)\right), &
\end{array}\right.
$$

and $r=r\left(\xi_{1}, \xi_{2}\right)=0.7+\frac{\xi_{1}+\xi_{2}}{10}$. Then (5.1) has the exact solution

$$
u(x, \omega)=\max \left\{\left(|x|^{2}-r^{2}\right) e^{\xi_{1}(\omega)+\xi_{2}(\omega)}, 0\right\}^{2} .
$$

Note that the coincidence set $\{x \in D \mid u(x, \omega)=0\} \subset D$ varies with $\omega$. 


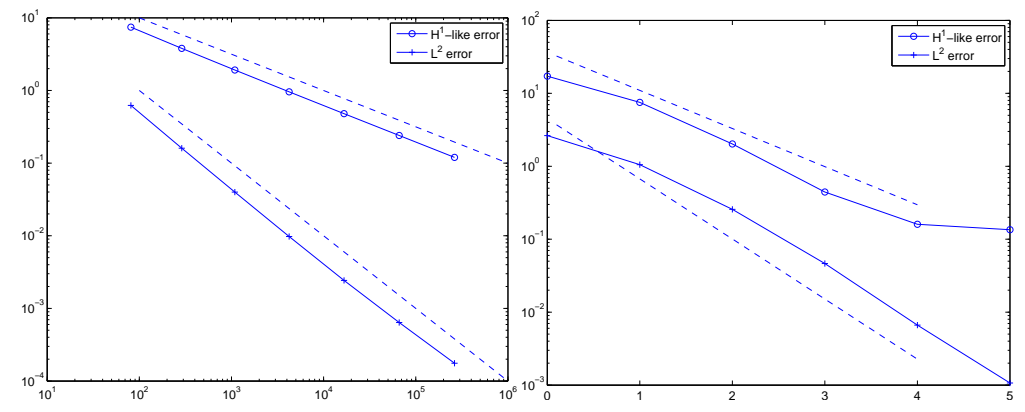

FiguRE 1. Discretization error in the $H^{1}$-like norm and the $L^{2}$ norm over the number of spatial unknowns $n_{j}$ for decreasing mesh size $h_{j}, j=2, \ldots, 8$, and fixed polynomial degree $m=5$ (left) and over increasing polynomial degree $m=0, \ldots, 5$, for fixed mesh size $h=h_{8}$ (right).

Replacing the random variables $\xi_{1}, \xi_{2}$ by parameters $y_{1}, y_{2}$ we obtain an equivalent deterministic obstacle problem on the parameter set $I=[-1,1] \times[-1,1]$ equipped with the induced measure $P_{\xi}=P_{\xi_{1}} P_{\xi_{2}}$ where $P_{\xi_{i}}=\frac{1}{2}$. The deterministic problem is discretized as described in Section 4.2. Here, we utilize a sequence of triangulations $\mathcal{T}_{j}$ with mesh size $h_{j}=2^{-j}$ as obtained by successive uniform refinement of an initial triangulation $\mathcal{T}_{0}$. The initial triangulation $\mathcal{T}_{0}$ is obtained by one uniform refinement step applied to a partition of $D$ into two congruent triangles. The associated finite element spaces with dimension $n_{j}=\left|\mathcal{N}_{j}\right|$ are denoted by $\mathcal{S}_{j}$. We use polynomial ansatz functions $\psi_{\nu_{1}}, \psi_{\nu_{2}}$ with common maximal degree $m_{1}=m_{2}=m$. The resulting discrete problems of the form (4.9) are solved by a truncated monotone multigrid method [15] with $V(3,3)$ cycle and nested iteration. We observed averaged convergence rates ranging from $0.08-0.12$.

In our first experiment we want to investigate how the discretization error depends on the mesh size $h_{j}$ and on the polynomial degree $m$. The left picture of Figure 1 shows the discretization error over the number of spatial unknowns $n_{j}$ for decreasing mesh size $h_{j}, j=2, \ldots, 8$, and fixed $m$. Here, the upper line with markers $\circ$ represents the $H^{1}$-like error $\left\|u-u_{\mathcal{S}_{j} \mathcal{Q}_{5}}\right\|$ behaving like $\mathcal{O}\left(h_{j}\right)$ (upper dashed line), while the lower line with markers $*$ shows the $L^{2}$ error $\left\|u-u_{\mathcal{S}_{j} \mathcal{Q}_{5}}\right\|_{0}$ which decreases with order $\mathcal{O}\left(h_{j}^{2}\right)$ (lower dashed line), as expected. In the right picture, we again display the discretization error in these two norms but now for fixed mesh size $h=h_{8}$ and increasing polynomial degree $m=0, \ldots, 5$. The upper line shows that the $H^{1}$-like error decreases like $\mathcal{O}\left(q^{m}\right)$ with $q=0.3$ (upper dashed line) until the spatial contribution is about to dominate the overall error for $m=5$. The lower line depicts the $L^{2}$ error behaving like $\mathcal{O}\left(q^{m}\right)$ with $q=0.15$ (lower dashed line).

We now evaluate the approximation of the expectation value $E[u]$ in a similar way. In the left picture of Figure 2 we observe that the error $\| E\left[u-u_{\mathcal{S} \mathcal{Q}]} \|_{L^{2}(D)}\right.$ behaves like $\mathcal{O}\left(h_{j}^{2}\right)$ (dashed line) for decreasing $h$ and fixed $m=5$, while the right picture indicates an exponential behavior like $\mathcal{O}\left(q^{2 m}\right)$ with $q=0.3$ for increasing $m$ and fixed $h=h_{8}$. Again the spatial error starts to dominate for $m \geq 4$. The experimental results displayed in Figure 1 and Figure 2 suggest possible extensions of discretization error estimates of the form (4.10) and $\left\|E\left[u-u_{\mathcal{S} \mathcal{Q}}\right]\right\|_{L^{2}(D)}=\mathcal{O}\left(\left\|u-u_{\mathcal{S} \mathcal{Q}}\right\|^{2}\right)$ from the linear case to stochastic obstacle problems. Theoretical justification will be the subject of further research.

In our final test, we want to compare the efficiency, i.e. the ratio of accuracy and corresponding computational effort, of the polynomial chaos approach to (5.1) and 


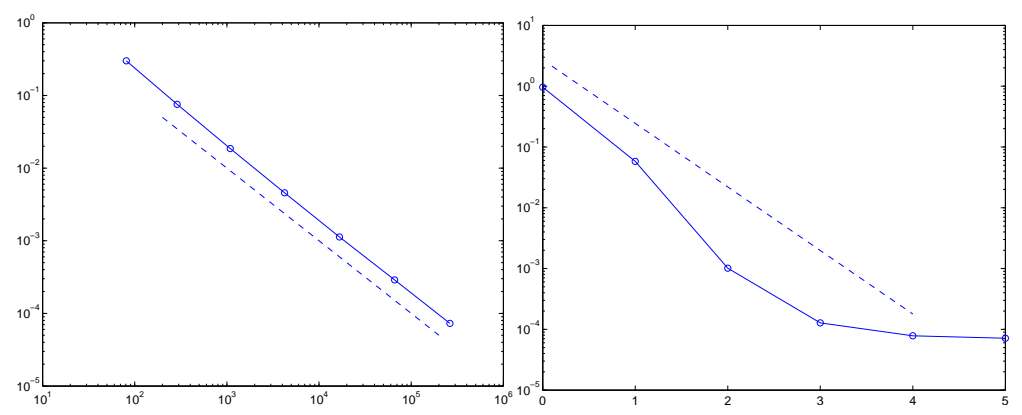

FIGURE 2. $L^{2}$ error of the expectation value over the number of spatial unknowns $n_{j}$ for decreasing mesh size $h_{j}, j=2, \ldots, 8$, and fixed polynomial degree $m=5$ (left) and over increasing polynomial degree $m=0, \ldots, 5$, for fixed mesh size $h=h_{8}$ (right).

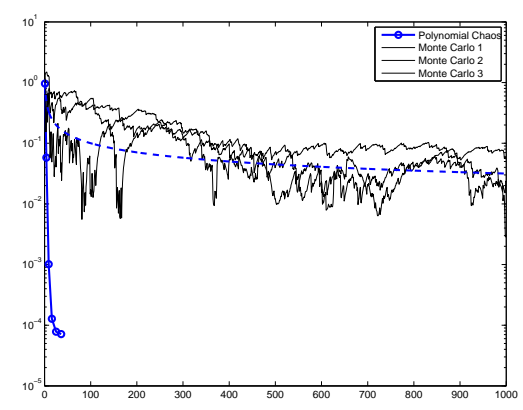

FiguRE 3. $L^{2}$ error of the expectation values over the number $N$ of deterministic solves: Monte Carlo runs (solid line), $\mathcal{O}(1 / \sqrt{N})$ (dashed line), and polynomial chaos (solid line with markers $\circ$ ).

of the Monte Carlo method. The solid lines in Figure 3 show the $L^{2}$ error of the expectation values for three different runs of the Monte Carlo method over the number $N$ of realizations, i.e. the number of deterministic spatial solves. The underlying mesh size is fixed to $h=h_{8}$. As expected, we observe that the error behaves like $\mathcal{O}(1 / \sqrt{N})$ (dashed line). On the other hand, we have a very fast error reduction of the polynomial chaos approach providing the almost vertical solid line (with markers o). The markers are associated with the polynomial degrees $m=0, \ldots, 5$. Observe that for $m=3$ the efficiency of the polynomial chaos approach is about 1000 times higher than for the Monte Carlo method. Obviously, this effect is due to the smoothness of $u$ which is exploited by polynomial chaos. Not so much can be gained for larger $m$ any more, because the spatial error starts to dominate the overall error.

5.2. A time discrete Richards equation. We consider the stochastic variational inequality 1.1 with $\phi$ generated by the piecewise smooth convex function

$$
\Phi(z)= \begin{cases}0.0458 z+0.2527\left((3.082 z+4.082)^{1.2252}-4.776\right), & -1.32446 \leq z \leq-1 \\ z, & -1<z,\end{cases}
$$

and choose a lognormal diffusion coefficient $K(x, \omega)$. The $\operatorname{logarithm} \log K(x, \omega)$ is approximated by a Karhunen-Loève expansion (3.3) of length $R=2$ with Gaussian random variables $\xi_{r}$, eigenvalues $\lambda_{r}$ and eigenfunctions $g_{r}$ of the exponential covariance kernel

$$
C_{K}(x, y)=e^{|x-y|_{1} / 40},
$$



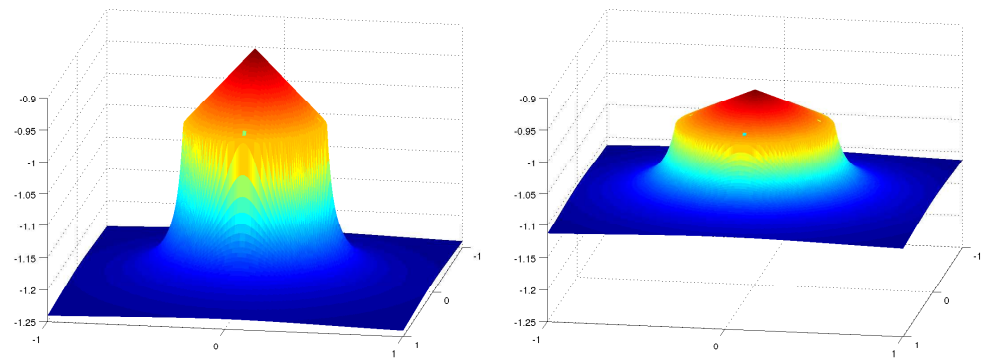

Figure 4. Exact solution $u(x, \omega)$ for $\left(\xi_{1}, \xi_{2}\right)=(0,0)$ (left) and $\left(\xi_{1}, \xi_{2}\right)=(0.5,-2.6)$ (right).

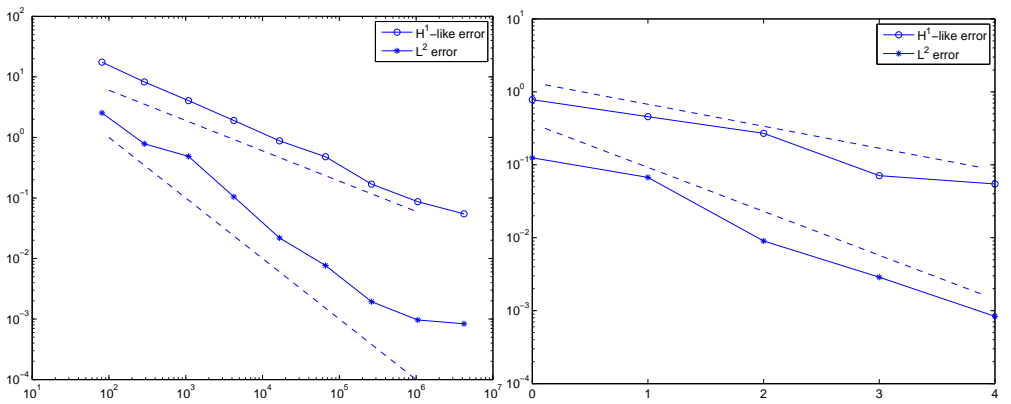

FiguRE 5. Discretization error in the $H^{1}$-like norm and the $L^{2}$ norm over the number of spatial unknowns $n_{j}$ for decreasing mesh size $h_{j}, j=2, \ldots, 10$, and fixed polynomial degree $m=4$ (left) and over increasing polynomial degree $m=0, \ldots, 4$, for fixed mesh size $h=h_{10}$ (right).

see [12, Section 2.3.3]. Again, we choose $D=(-1,1) \times(-1,1)$ and $l(v)=E\left[\int_{D} f v \mathrm{~d} x\right]$ where $f(x, \omega)$ is now selected such that

$$
u(x, \omega)=\left\{\begin{array}{cl}
-1+0.05(r-|x|) . & |x|<r \\
\left(\xi_{1}^{4}(\omega)-\left(3-\xi_{1}(\omega)\right)^{2}-3 \xi_{1}(\omega)+15\right) \cos ^{2}\left(\xi_{2}(\omega) \pi / 2\right), & |x| \geq r \\
-1-\left(2\left|\xi_{1}(\omega)+\xi_{2}(\omega)\right|+4\right)^{-1}+(100(|x|-0.36))^{-1}, & |x| \geq
\end{array}\right.
$$

with $r=r\left(\xi_{1}, \xi_{2}\right)=0.4+0.02\left|\xi_{1}+\xi_{2}\right|$ is the exact solution of the corresponding stochastic variational inequality (1.1). The solution $u(x, \omega)$ is illustrated in Figure 4 Related problems typically arise from time discretization of Richards equation modeling saturated/unsaturated groundwater flow, cf., e.g., [5]

A parameteric deterministic formulation (3.10) is obtained by replacing the random variables $\xi=\left(\xi_{1}, \xi_{2}\right)$ by coordinates $\mathbf{y}=\left(y_{1}, y_{2}\right) \in I=\mathbb{R}^{2}$. The probability density $P_{\xi}=\operatorname{pdf}_{2}\left(y_{1}\right) \operatorname{pdf}_{2}\left(y_{2}\right)$ on $I$ is obtained from the standard normal distribution $\operatorname{pdf}_{i}\left(y_{i}\right)=(2 \pi)^{-1 / 2} \exp \left(-y_{i}^{2} / 2\right)$. The deterministic problem is discretized as described in Section 4.2 based on the same sequence of triangulations $\mathcal{T}_{j}, j=0, \ldots, 10$, as in the preceding section. Again, we use polynomial ansatz functions $\psi_{\nu_{1}}, \psi_{\nu_{1}}$ with common maximal degree $m_{1}=m_{2}=m$. The resulting discrete problems of the form (4.9) are solved by a truncated monotone multigrid method 18] with $V(3,3)$ cycle and nested iteration. We observed averaged convergence rates ranging from $0.80-0.87$.

Figure 5 displays how the discretization error depends on the mesh size $h_{j}$ and on the polynomial degree $m=0, \ldots, 4$. As in the previous example, the left picture shows 


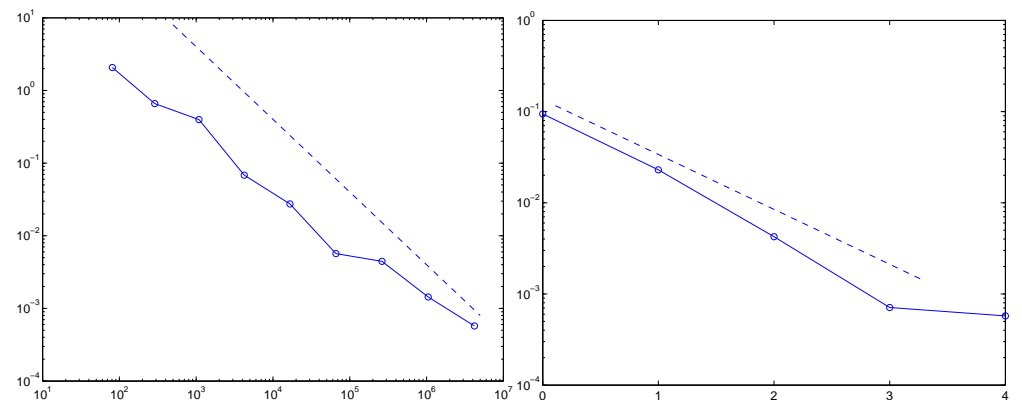

FiguRE 6. $L^{2}$ error of the expectation value over the number of spatial unknowns $n_{j}$ for decreasing mesh size $h_{j}, j=2, \ldots, 10$, and fixed polynomial degree $m=5$ (left) and over increasing polynomial degree $m=0, \ldots, 5$, for fixed mesh size $h=h_{10}$ (right).

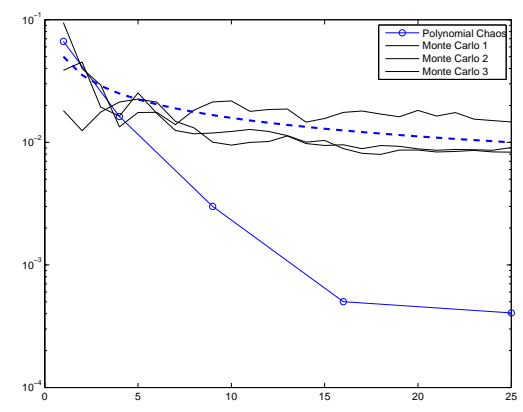

FiguRe 7. $L^{2}$ error of the expectation values over the number $N$ of deterministic solves: Monte Carlo runs (solid line), $\mathcal{O}(1 / \sqrt{N})$ (dashed line), and polynomial chaos (solid line with markers $\circ$ ).

that for fixed polynomial degree $m=4$ and decreasing mesh size $h_{j}, j=2, \ldots, 10$, the $H^{1}$-like error $\left\|u-u_{\mathcal{S}_{j} \mathcal{Q}_{4}}\right\|$ (upper solid line with markers o) decreases with order $\mathcal{O}\left(h_{j}\right)$ (upper dashed line) while the $L^{2}$ error $\left\|u-u_{\mathcal{S}_{j} \mathcal{Q}_{4}}\right\|_{0}$ (lower solid line with markers $*$ ) behaves like $\mathcal{O}\left(h_{j}^{2}\right)$ (lower dashed line). For fixed mesh size $h=h_{10}$ and increasing polynomial degree $m=0, \ldots, 4$. the right picture of Figure 5 shows an exponential decay of the $H^{1}$-like error (upper solid line with markers $\circ$ ) and of the $L^{2}$ error (lower solid line with markers $*$ ) of order $\mathcal{O}\left(q^{m}\right)$ with $q=0.5$ and $q=0.25$, respectively (dashed lines).

The $L^{2}$ error of the expectation value $E[u]$ is investigated in a similar way. The left picture of Figure 6 indicates that $\left\|E[u]-E\left[u_{\mathcal{S}_{j} \mathcal{Q}_{m}}\right]\right\|$ behaves like $\mathcal{O}\left(h_{j}^{2}\right)$ (dashed line) for decreasing mesh size $h_{j}$ and fixed $m=4$ while the right picture shows an exponential decay of order $\mathcal{O}\left(q^{2 m}\right)$ with $q=0.5$ (dashed line) for increasing $m$ and fixed $h=h_{10}$. Note that the spatial error starts to dominate for $m \geq 3$. These experimental results also suggest discretization error estimates of the form 4.10) and $\left\|E\left[u-u_{\mathcal{S} \mathcal{Q}}\right]\right\|_{L^{2}(D)}=\mathcal{O}\left(\left\|u-u_{\mathcal{S} \mathcal{Q}}\right\|^{2}\right)$ for stochastic variational inequalities of the second kind.

In order to compare the polynomial chaos approach with the Monte Carlo method, we consider the fixed mesh size $h=h_{10}$. Figure $\square$ then displays the $L^{2}$ errors of three runs of the Monte Carlo method (upper solid lines) and the expected $\mathcal{O}(1 / \sqrt{N})$ behavior (dashed line) together with the $L^{2}$ error of the polynomial chaos approach (lower line with markers $\circ$ ) over the number $N$ of deterministic solves. In contrast to the previous section, we bound the maximal number of deterministic solves by 
$\left|\mathcal{Q}_{4}\right|$. Similar to the previous example, we observe a much higher efficiency of the polynomial chaos approach. Here, the factor is about 20 for $m=3$, because the spatial error does not allow for higher accuracy. Again, this is a consequence of the smoothness of $u$ which is exploited by polynomial chaos and not by Monte Carlo.

\section{REFERENCES}

[1] Ivo Babuška and Panagiotis Chatzipantelidis. On solving elliptic stochastic partial differential equations. Comput. Methods Appl. Mech. Eng., 191:40934122, 2002.

[2] Ivo Babuška, Raúl Tempone, and Georgios E. Zouraris. Galerkin finite element approximations of stochastic elliptic partial differential equations. SIAM J. Num. Anal., 42(2):800-825, 2004.

[3] Ivo Babuška, Raúl Tempone, and Georgios E. Zouraris. Solving elliptic boundary value problems with uncertain coefficients by the finite element method: the stochastic formulation. Comput. Methods Appl. Mech. Engrg., 194(12-16): 1251-1294, 2005.

[4] Ivo Babuška, Fabio Nobile, and Raúl Tempone. A stochastic collocation method for elliptic partial differential equations with random input data. SIAM J. Num. Anal., 45(3):1005-1034, 2007.

[5] Heiko Berninger, Ralf Kornhuber, and Oliver Sander. Fast and robust numerical solution of the Richards equation in homogeneous soil. Preprint Pr-A-10-01, FU Berlin, 2010.

[6] Haiyan Cheng and Adrian Sandu. Uncertainty quantification and apportionment in air quality models using the polynomial chaos method. Envir. Model. ES Softw., 24:917-925, 2009.

[7] Albert Cohen, Ron DeVore, and Christoph Schwab. Analytic regularity and polynomial approximation of parametric and stochastic elliptic pdes. Research report no. 2010-03, ETH Zürich, 2010.

[8] Manas K. Deb, Ivo M. Babuška, and J. Tinsley Oden. Solution of stochastic partial differential equations using Galerkin finite element techniques. Comput. Methods Appl. Mech. Engrg., 190:6359-6372, 2001.

[9] Ivar Ekeland and Roger Témam. Convex Analysis and Variational Problems. Classics in Applied Mathematics. SIAM, 1999. Reprint.

[10] Ralf Forster. On the stochastic Richards equation. PhD thesis, Freie Universität Berlin, in preparation.

[11] Philipp Frauenfelder, Christoph Schwab, and Radu A. Todor. Finite elements for elliptic problems with stochastic coefficients. Comput. Methods Appl. Mech. Engrg., 194(2-5):205-228, 2005.

[12] Roger G. Ghanem and Pol D. Spanos. Stochastic Finite Elements: A Spectral Approach. Springer, 1991.

[13] Roland Glowinski. Numerical methods for nonlinear variational problems. Springer, 1984.

[14] Roland Glowinski, Jacques-Louis Lions, and Raymond Trémolières. Numerical Analysis of Variational Inequalities. North-Holland, Amsterdam, 1981.

[15] Carsten Gräser and Ralf Kornhuber. Multigrid methods for obstacle problems. J. Comput. Math., 27:1-44, 2009.

[16] Kari Karhunen. Über lineare Methoden in der Wahrscheinlichkeitsrechnung. Ann. Acad. Sci. Fennicae, Ser. A. I., 37:1-79, 1947.

[17] George Em Karniadakis, Chau-Hsing Su, Dongbin Xiu, Didier Lucor, Christoph Schwab, and Radu A. Todor. Generalized polynomial chaos solution for differential equations with random inputs. Research report no. 2005-01, ETH Zürich, 2005. 
[18] Ralf Kornhuber. On constrained Newton linearization and multigrid for variational inequalities. Numer. Math., 91:699-721, 2002.

[19] Olivier P. Le Maître and Omar Knio. Spectral Methods for Uncertainty Quantification. Springer, 2010.

[20] Olivier P. Le Maître, Omar M. Knio, Habib N. Najm, and Roger G. Ghanem. A stochastic projection method for fluid flow: I. Basic formulation. J. Comp. Phys., 173(2):481-511, 2001.

[21] Olivier P. Le Maître, Matthew T. Reagan, Habib N. Najm, Roger G. Ghanem, and Omar M. Knio. A stochastic projection method for fluid flow: II. Random process. J. Comp. Phys., 181(1):9-44, 2002.

[22] Michel Loève. Probability Theory. D. Van Nostrand Company, 3rd edition, 1963.

[23] Bernt Øksendal. Stochastic Differential Equations. Springer, 6th edition, 2007.

[24] Carl Philip Rupert and Cass T. Miller. An analysis of polynomial chaos approximations for modeling single-fluid-phase flow in porous medium systems. J. Comp. Phys., 226:2175-2205, 2007.

[25] Christoph Schwab and Radu A. Todor. Karhunen-Loève approximation of random fields by generalized fast multipole methods. J. Comp. Phys., 217(1): 100-122, 2006.

[26] Radu A. Todor and Christoph Schwab. Convergence rates for sparse chaos approximations of elliptic problems with stochastic coefficients. Research report no. 2006-05, ETH Zürich, 2006.

[27] Norbert Wiener. The homogeneous chaos. Am. J. Math., 60:897-936, 1938.

[28] Dongbin Xiu and Jan S. Hesthaven. High-order collocation methods for differential equations with random inputs. SIAM J. Sci. Comput., 27(3):1118-1139, 2005.

Ralf Forster, Freie Universität Berlin, Institut für Mathematik, Arnimallee 6, 14195 Berlin, GERMANY

E-mail address: forster@math.fu-berlin.de

Ralf Kornhuber, Freie Universität Berlin, Institut für Mathematik, Arnimallee 6, 14195 Berlin, Germany

E-mail address: kornhuber@math.fu-berlin.de 